\title{
FLIPPING A 700 STUDENT CLASSROOM
}

\author{
Peter Purgathofer, \& Kay Kender \\ HCI Research Department, Faculty of Informatics, TU Wien (Austria)
}

\begin{abstract}
Because of the pandemic, we had to switch our first-semester informatics course with 700 students from lecture hall teaching to online teaching. We used the opportunity to rethink our approach and come up with forms of teaching that fit the circumstances. In the evaluation, we found that students generally appreciated our efforts, and although we raised the requirements for a positive grade, the share of students who passed the course was higher than usual.
\end{abstract}

Keywords: Flipped classroom, university, large course, experiences, feedback.

\section{Context and motivation}

While the origins of the flipped classroom concept are hard to pin down, we can attribute widespread academic attention to this concept to around 2012, when a number of scientific conferences were dedicated to this idea, e.g. the first ICM Conference in Munich. The flipped classroom is, in short, defined as »events that have traditionally taken place inside the classroom now take place outside the classroom and vice versa (Lage, Platt \& Treglia, 2000). In a setting like the one we experience at TU Wien, where roughly 700 students are admitted into the bachelor programs of the faculty of informatics, little of what happens outside the classroom, or rather, the largest lecture hall of TU Wien, is under our control. Consequently, flipping the classroom was never something we considered, focusing instead on making the lectures as good as possible under the circumstances.

COVID-19 made us reconsider when we were forced to leave the lecture hall behind. Moving our lectures to an online format without losing essential aspects of a physical lecture hall was impossible: hundreds of students in the same room at the same time, interacting before, during, and after the lecture, the possibility to »feel the crowd«, the joy of physical presence, the commitment to a shared experience, and other facets of classroom lectures had to be left behind as we were all confined behind private screens in small rooms.

We had to explore new forms of teaching as we redesigned our course to create an adequate learning environment in a mode of operation where physical presence was impossible. This paper describes our approach in this redesign, presents its result, offers a glimpse into the evaluation of our reorganization, and discusses our conclusions and further plans.

\section{Relevant related research}

The concept of the flipped or inverted classroom has seen a lot of academic attention lately. While in August 2019, a search of Google Scholar yielded »approximately 70,000 and 60,000 hits for the

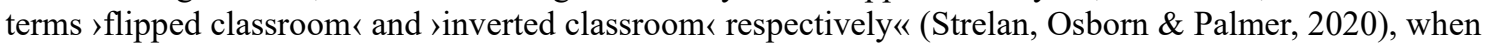
this article was written in May 2021, these numbers had risen to 120,000 and 90,000. This heightened attention can at least partially be attributed to the necessities of teaching during a pandemic, with many scholars forced to rethink their approaches to teaching.

Strelan et al. (2020) give an excellent overview over the existing research regarding the effectiveness of flipping the classroom in the form of a meta-analysis of nearly 200 studies. They conclude that »Overall, flipping a classroom has a positive, moderate effect on student performance «. In our view, their core finding is that »what distinguishes a flipped classroom from traditional teaching is not so much that information is pre-recorded [...] but, rather, that students have an opportunity to engage in active learning and problem-solving with hands-on guidance provided by an expert.«.

Active, self-determined and responsible learning has long been a goal in our efforts to redesign our exercises. The pandemic gave us the incentive to rethink our lectures completely. 


\section{Our approach}

Before the changes made necessary by the pandemic, our course »Ways of thinking in informatics « (Frauenberger \& Purgathofer, 2019) was a rather traditional, if very large, university course. We held lectures twice a week for 135 minutes in the largest lecture hall of TU Wien, offering a detailed treatment of the course content. This was complemented with exercises for some of the chapters, where students had to do their own research, read literature, and produce a research paper of sorts describing their work and results. Of the nine chapters of our lectures, six were essential enough to warrant such exercises. These exercises are organised in a way that requires students to review the work of their peers (Luckner \& Purgathofer, 2015), using a platform we have been developing for over ten years now (Luckner, Purgathofer \& Fitzpatrick, 2019). Towards the end, the course required the students to discuss a problem in small groups and hand in a paper documenting their individual positions as well as the results of the discussion.

The pandemic removed the lecture hall from the equation. Since our course is held in the winter term, we had the chance to hear from students who had witnessed direct online transformation models in other large lectures during the previous semester, when lockdowns first began. While students generally praised the flexibility and availability of recorded lectures, online lectures of equal length as classroom lectures were perceived as exhausting and stressful. This experience was shared by many lecturers, who describe the experience as »talking into the void« and »losing all contact to students«. Drawing from these anecdotes, we decided that the one thing we did not want to do were 135 minute zoom lectures.

Another aspect we considered was that the »Ways of thinking in informatics « course is designated for the first semester. The 2020 winter term was thought to be the first time first-semester students would come to our university without meeting each other. Usually, they meet at lecture halls, seminar rooms and other workspaces, as well as cafeterias and canteens. But this year, they would only meet online, and most of these online meetings barely deserve the term »meeting« at all.

\subsection{Goals}

Based on these thoughts and observations, we came up with a catalogue of goals. We wanted students to experience our course as different from the other courses in a positive way. We wanted to give them an opportunity to talk to each other in a social setting, guided by our course content. We wanted to encourage them to ask questions about the course content, the organization of the lecture, and about how what they learn in the course relates to the rest of informatics, and the world around them. We wanted students to have experiences in the online course that feel less distanced than the rather anonymous lecture hall version, even if they are at home or in their dorm room. We wanted to use the possibilities technology offers us to do things we had never done before. And finally, we wanted to offer students a variety of ways to access the course content.

The exercises and the way they are organized and graded were to remain largely unchanged. We raised the requirements a little, adding a task to each chapter in which students had to produce a summary of the chapter's topic as presented. The goal of this assignment was to establish better connections between lectures and exercises. We decided to leave this part mostly untouched, because the exercise part that was already working well in an online format (Luckner \& Purgathofer, 2015), and since we were planning to change so many things, we had no resources to completely rethink it. In other words, the exercise component formed the basis for our reorganization.

\subsection{Building blocks}

The following formats were devised to implement our goals as efficiently as possible in the given timeframe, with the available means:

$\rightarrow$ We set up a little production/streaming studio that enabled us to create a video format where the lecturer was part of the presentation. We did this to have control over the position of the lecturer in the streamed or recorded content, so that facial expression and gestures could be captured regardless of the students' setup. With this setup, we were able to create several different presentation layouts and make them available to lecturers.

$\rightarrow$ We developed a community-moderated Q\&A routine, based on the format of the Office Hour by Alex Lindsay, described by Daulton (2020), using the Q\&A-tool of Zoom webinars. In a Zoom webinar, only explicitly invited participants (panelists) share their video, while the rest (audience) can watch video and audio streams. All participants can propose questions using Zoom's built-in Q\&A-tool, see the questions proposed by others, and »upvote« the questions they deem interesting. That way, a list of questions ordered by audience interest is available to the panel. A »question moderator « on the panel reads the top-rated question from this list out for the panelists to discuss or answer. This is repeated until either time runs out or all questions are answered. As all questions are available as plain text, they can be 
downloaded after the session. By tracking the time code of when each question asked, we were able to create an indexed list of questions that enabled students to navigate to a specific answer in the videos.

$\rightarrow$ The bi-weekly 135 minute lecture format was replaced by short 30 to 45 minute online introductory lectures that provided an overview over each chapter. The Zoom sessions for these were planned to take an hour so the remaining time could be dedicated to answering comprehension questions asked by the students. For this, we used the community-moderated Q\&A-format described above, with the lecturer and the question moderator as the only panelists. These introductory sessions were recorded.

$\rightarrow$ All the additional content for each week was prepared in a format we called SlideBooks, which was developed by a graduate student (Kuroll, 2020). SlideBooks are created from the presentation slides using automation, based on a simple syntax of commands that are embedded in the speaker notes. In their final form, they combine images and text from the slides with additional text, as well as links to sources and external resources from the speaker notes. SlideBooks can be commented on by students on a slide-by-slide basis. Most SlideBooks were made from the slides of the respective introductory lectures, but expanded with text and links from the speaker notes to contain approximately double the information from the lecture.

Figure 1. Section from a SlideBook, showing three slides with different layouts, additional links and comments.
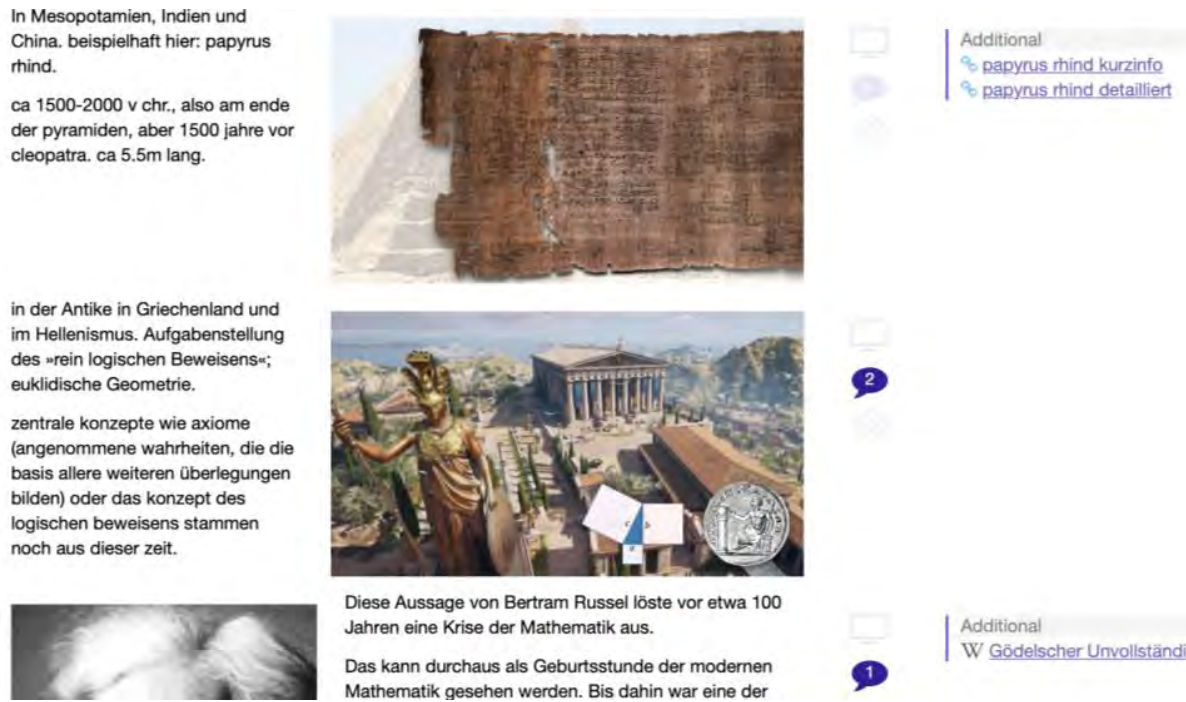

| Adiditional

Diese Aussage von Bertram Russel löste vor etwa 100 Jahren eine Krise der Mathematik aus.

Das kann durchaus als Geburtsstunde der modernen

$\rightarrow$ Additionally, we produced short video clips for content that detailed or expanded on the content of the introductory lectures. These videos were produced using the same setup as the lecture itself, a talking head on top of the slides. Eighteen videos of this type were produced over the semester, with an average length of 15 minutes.

$\rightarrow$ To enable social interaction centered in the content of our course, we required students to partake in an online interactive session every other week, held via Zoom. We asked students to work on specific assignments in groups of ten to fifteen people in breakout rooms. The assignments were derived from the content of the course, and were specifically designed not to be too difficult, so that the conversations would be stress-free and casual. We used platforms like miro.com to facilitate interaction. As an example of such a session, in a chapter focused on responsibility and ethics, students discussed the Chinese social credit system from various perspectives. To protect the students' privacy, these sessions were not recorded.

$\rightarrow$ Our community-moderated Q\&A format was the basis of the discussion sessions. Here, we invited external experts for the topics at hand to discuss questions provided by the students. We usually had between two and three experts on the panel, and additionally invited students who volunteered to the panel. With a thematic moderator and the question moderator, those sessions had between five and ten panelists, answering and discussing whatever came from the students via the Q\&A-tool. Those sessions were recorded and could be watched later, where the indexed list of questions made it possible to easily navigate to selected parts of the discussion.

\subsection{Structure and progress}

Starting October 2020, the course was held using the building blocks described above. Each of the nine chapters began with an introductory lecture (usually Monday), followed by an interactive session a couple of days later (usually Wednesday). Longer chapters featured a second introductory 
lecture. Each chapter would end with a discussion session (usually on Thursday). Additional materials like SlideBooks and video clips were made available starting with the introduction of each chapter, with all materials available by the time of the discussion. Six of the chapters had exercises associated with them that were published on the day after the discussion session, with a two-week deadline. Other than the interactive sessions and the hand-in of assignments, no format was mandatory to attend or watch.

\section{Methodology, evaluation \& discussion}

At the very end of every semester, we ask all students for feedback about their experiences attending the course. Last winter term, students answered six questions, writing an average of roughly 3.000 ( $\min 700$, max 9.000) characters. 497 completed and handed in feedback, of which 170 were chosen randomly as a basis for this evaluation.

One of the questions specifically asked for feedback and suggestions regarding the new elements: introductory lectures, interactive sessions, discussion sessions, SlideBook, and additional videos. We conducted a mixed qualitative/quantitative evaluation of these answers, using a variant of sentiment analysis (Feldman, 2013) to extract an understanding of the overall satisfaction with our format. In this analysis, we assigned each statement an integer value between 1 (very positive) and 5 (very negative) in relation to the aspect evaluated against.

Figure 3. Distribution of sentiment towards the different formats. All graphs use the same scale of absolute numbers.
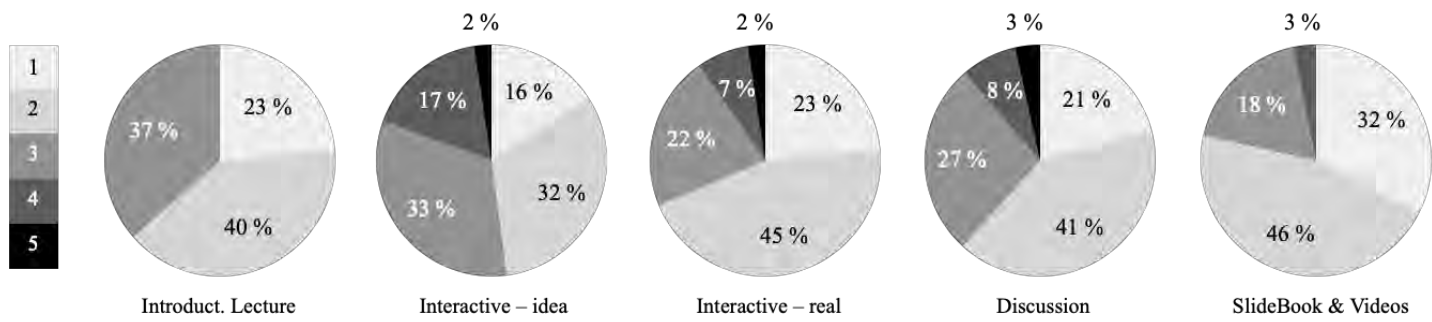

\subsection{Introductory lectures}

$\rightarrow$ Introductory lectures were watched by more than $85 \%$ of our students. $23 \%$ of these students positively mentioned the possibility to ask questions after the lecture part. The average sentiment towards the introductory lectures by the students who used them was 2.1., with no student having negative sentiment (4 or 5) towards this element.

Among the aspects attributed to this positive rating were that the duration was appropriate, especially compared to other courses, and that questions could be asked at the end. Criticism was offered regarding the low resolution of the recording (this was solved during the semester), and students wanted to play back the recordings faster than real-time (also solved during the semester). One student found our presentation »too informal«, and asked us to cut the occasional chit-chat at the beginning of lecture units, while others favourably mentioned that the informal tone made us appear more approachable.

$\rightarrow$ We experienced some technical difficulties in the organization of interactive sessions. Contrary to the documentation, Zoom did not allow us to open enough breakout rooms, so that the group sizes were larger than expected. Creating the breakout rooms turned out to be an iterative process, where we had to start with a too-high number of rooms and slowly reduce it until it worked. These problems were noted by many students as distracting and annoying. A frequent conclusion was that they liked the concept, but didn't like the implementation.

Because of the technical difficulties, our evaluation differentiated between the idea and the implementation of the interactive session. The average sentiment of the students toward the idea was quite positive with 2.2, while the actual implementation was criticized more, with a resulting value of 2.6. Figure 3 shows the distribution of sentiments here.

One of the goals of the interactive sessions was to create an environment for students to meet colleagues and socialize in the context of a course. As these sessions were mandatory and not recorded, all students had to participate in person. $18 \%$ of the students stated that they used the format as an opportunity to get in touch and interact with their new colleagues. On the other hand, $25.9 \%$ of students reported incidents where colleagues disrupted their work with immature »trolling «, or went »dark « during the group work phases. Both problems indicate that some students did not take this format seriously enough, as we only required their attendance, not contributions. Still, one student wrote (translated from German): »Especially during the pandemic it was my only chance to talk to and collaborate with other students, and thus I have met my first university friend «. 
$\rightarrow$ The discussion sessions were voluntary, we found a significantly lower interest in them. Only $68 \%$ of students attended or watched discussions. On average, their rating was positive, with a sentiment average of 2.3, but opinions were strong: while some students rated the discussions as completely useless, others described them as their favourite part of the course, especially when the guests were interesting. Some students wrote that the written Q\&A is too cumbersome, especially when you want to listen to the discussion and at the same time write a question. Others mentioned that the written question format was especially suitable for a discussion with so many participants.

Our own observation is that the format offered a couple of unique advantages over lecture hall discussions. One obvious advantage is that for the guests, it is time-saving and generally much easier to participate in an online discussion than come to a lecture hall. We were able to invite German-speaking guests from all over Europe. The written questions were also an advantage. This effectively made it impossible to ask long and rambling questions, and it meant that questions were generally more focused and precise. Also, there were less inhibitions to write and submit a question compared to standing up and asking a question in front of a full lecture hall. As a result, a broader variety of questions were asked, and we presume that some students asked questions who never would have spoken out in a lecture hall. Finally, the community ranking makes it possible for students to ask questions tentatively, leaving the community to decide whether a question is worth asking, rather than self-censoring. Consequently, some of the most interesting, weird and challenging questions we have ever heard were discussed.

$\rightarrow$ SlideBook has seen the best reception among our new formats. With an average sentiment of 1.9 - see Figure 3 for more details - no other format was praised so much for its value. Students emphasized the structure and organization, which makes access to individual topics much quicker than searching in videos. They also highlighted that a text-based format leaves the choice of pace to the learner.

$\rightarrow$ Strangely enough the additional videos were not seen as such, but as an alternative to the SlideBook content by most students. This can partly be attributed to a problem in presentation, but probably also goes back to a conflict in media semantics. In the case of introductory lectures, SlideBooks were offered as a different representation of the same content, although SlideBooks contained videos that extended this content in other places.

\section{Conclusions}

Our evaluation supports the understanding that the concepts and formats we deployed were welcomed by students. The extensive textual feedback from the students gives us plenty of starting points to improve on, but we end our discussion here with the result, as we have run out of space. In the end, the share of students that passed the course was slightly higher than average, compared to recent years. This is especially interesting since, in many other courses at our university, the number was much lower than usual this year. We take that as an affirmation of our approach.

\section{References}

Daulton, Doug. (2020) Office Hours with Alex Lindsay. Retrieved May 24, 2020, from https://focusonmobile.com/2020/05/01/office-hours-with-alex-lindsay-and-friends

Feldman, R. (2013). Techniques and applications for sentiment analysis. Communications of the ACM, 56(4), 82-89.

Frauenberger, C., \& Purgathofer, P. (2019). Ways of thinking in informatics. Communications of the ACM, 62(7), 58-64

Lage, M., Platt, G., Treglia, M. (2000). Inverting the Classroom: A Gateway to Creating an Inclusive Learning Environment- The Journal of Economic Education 31, Nr. 1: 32.

Luckner, N., \& Purgathofer, P. (2015). Exploring the use of peer review in large university courses. IxD\&A, 25, 21-38.

Luckner, N., Purgathofer, P., \& Fitzpatrick, G. (2019). Involving Students in the Co-Creation of a Complex. Evolving Learning Environment. IxD\&A, 42, 70 - 92.

Strelan, P., Osborn, A., \& Palmer, E. (2020). The flipped classroom: A meta-analysis of effects on student performance across disciplines and education levels. Educational Research Review, 30, 100314. 